\title{
Knowledge coevolution: generating new understanding through bridging and strengthening distinct knowledge systems and empowering local knowledge holders
}

\author{
J. M. Chapman ${ }^{1}$ iD $\cdot$ S. Schott ${ }^{2}$
}

Received: 8 July 2019 / Accepted: 7 January 2020 / Published online: 28 January 2020

(c) The Author(s) 2020

\begin{abstract}
The effective and appropriate bridging of Western science with traditional or Indigenous knowledge is an ongoing discussion in the literature and in practice. The discourse transitioned from separate knowledge system to knowledge integration and most recently to knowledge co-production. We argue it is the moral and ethical responsibility of Western scientists working in and with Indigenous communities to make a concerted effort to collectively create mutually advantageous new knowledge while strengthening traditional knowledge and considering the normative impacts of Western science methods. Our knowledge coevolution framework provides guidance for achieving this in a flexible manner that can be applied to an array of research programs. Project governance structure, steps for implementation, checks and balances, and challenges are presented within the context of research project execution. We then illustrate application of the model throughout a harvest study conducted in Gjoa Haven, Nunavut, Canada.
\end{abstract}

Keywords Co-management $\cdot$ Research governance $\cdot$ Indigenous knowledge $\cdot$ Knowledge co-production $\cdot$ Selfdetermination

\section{Introduction}

The Supreme Court of Canada ruled that Indigenous communities holding official land title have a right to benefit from the land economically and determine how that land will be used by future generations (Tisljqot'in Nation v. British Columbia, 2014). Legacy resource management structures that assert federal and provincial jurisdiction over Indigenous people's access and control over natural resources within officially recognized settlement areas are slowly being amended to account for their right to self-determination. At the same time, major efforts have been put forth to achieve reconciliation in Canada, leading to a strengthened movement of Indigenous cultural revitalization and accelerated progress

Handled by Alexander Gonzalez Flor, University of the Philippines Open University, Philippines.

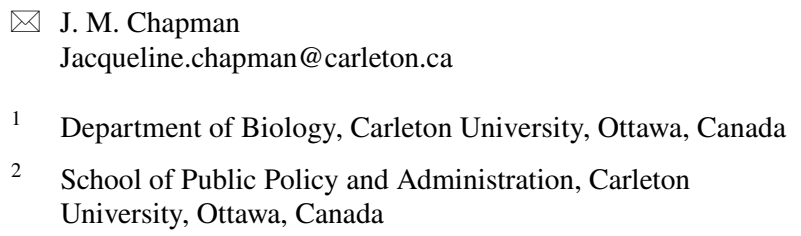

2 School of Public Policy and Administration, Carleton University, Ottawa, Canada

towards self-governance and determination. Through this process, resource management is supposed to be transferred back to Indigenous communities across Canada, but implementation strategies remain a challenge, and may be further slowed by uncertainty. For example, in the Arctic Inuit Settlement Region of Nunavut, policy for fisheries management is not based on the co-management structure implemented by the Nunavut Lands Claim Agreement Act (1993), but instead still relies on outdated Northwest Territories (NWT) Legislation (Boudreau and Fanning 2016; Suluk and Blakney 2008). In July 2018, the Department of Fisheries and Oceans and the Government of Nunavut (Fisheries and Oceans Canada 2018) released a statement indicating a restructuring of fisheries management in the region to formalize a co-management structure for fisheries development and exploitation.

Evidence-based policy and management practices are now the so-called "gold standard" of sustainable resource management. 'Evidence' in this context is typically derived from empirical Western science (WS), but the inclusion of Indigenous knowledge systems is becoming more common as reconciliation and reclamation of rights progresses. Knowledge held by Indigenous groups is often termed Traditional Knowledge (TK), while TK that is relevant 
for conservation science and wildlife management is often referred to as Traditional Ecological Knowledge (TEK). TEK is more increasingly included in environmentally based research, particularly research occurring on traditional territories associated with culturally important regions or species (Kimmerer and Lake, 2001; Nadasdy 2003; Gondor 2016; Abu et al. 2019). This inclusion, however, may disproportionately benefit researchers and their government or academic institutions rather than communities; indeed the 'decolonization' of research to break the inherent power dynamics of knowledge co-production and comanagement is a topic that has been discussed more widely in recent years (Nadasdy 2003; Marsh et al. 2015; Johnson et al. 2016). While non-Indigenous researchers often recognize this imbalance (Lowan-Trudeau 2012), there is little support from funding agencies and regulatory bodies in place to facilitate the deconstruction and redistribution of benefits associated with research programs. Recently, the National Inuit Strategy on Research released by Inuit Tapiriit Kanatami in 2018 describes research as a tool of colonialism where Inuit are historically either subjects or bystanders, ignoring their legal rights of self-determination (ITK 2018). The strategy identifies five priority areas that if implemented create a foundation from which researchers can support and facilitate Inuit self-determination for research occurring in Inuit Nunangat (all four Inuit settlement regions in Canada) by focusing on increased involvement of TK, empowerment, and ownership of research outcomes.

However, challenges arise when considering how to properly bridge Western and Indigenous knowledge in research in a manner that is relevant for co-management. Co-management relies on a distribution of power and the sharing of knowledge and data between authorities in the management and governance of natural resources (Berkes 2009). Unequal power dynamics often force TK holders to make difficult decisions regarding the sharing of their knowledge as they decide whether or not they are willing to risk having their knowledge misused and appropriated by the dominant actors, or be left out of the process all together (Von der Porten et al. 2016). Knowledge integration remains a dominant perspective in environmental governance, although the approach has been widely criticized by scholars for its failure to adequately address the role of unequal power dynamics, ongoing colonial tendencies, land-theft, and centuries of broken promises in environmental negotiations. Nadasdy (1999) and Roué and Nakashima (2002) have criticized knowledge integration on the basis that it is a one-way track, and the epistemology which TK is being integrated into holds the power to decide what is relevant and what knowledge system is used to validate new knowledge (Tengö et al. (2014)).

Challenges of the knowledge integration process have led scholars to shift in recent years toward a knowledge coproduction model which refers to "a process of bringing a plurality of knowledge sources and types together to address a defined problem and build an integrated or systems-oriented understanding of that problem" (Armitage et al. 2011). This method is inherently transdisciplinary and joins unique knowledge systems, observations, and fact derivations to provide a holistic understanding of a problem (Armitage et al. 2011). "Knowledge integration" is often, however, one component or step of the "knowledge co-production" process. Integrating TK can be considered controversial as it could either lead to knowledge appropriation, a loss of some aspects of cultural identity or knowledge transfer capacity, or tokenism - the inclusion of TK with the sole purpose of benefitting the researchers by appeasing formal requirements or to simply 'look good' among peers. Knowledge co-production is a generally vague term that often does not focus on preserving knowledge systems and cultural interpretations of knowledge claims. Each knowledge co-production process, therefore, needs to define the extent to which a knowledge type should be bridged with another. Rathwell et al. (2015) provide a typology of settings and examples how knowledge can be bridged. Knowledge integration might be useful and necessary in certain facets as long as it advances the application of knowledge towards a benefit for TK holders that have the most to lose. Achieving tangible outcomes for TK holders might, however, come with the cost of TK deterioration, which in turn increases power imbalances, and counteracts two-way sharing of knowledge and data in a respectful and effective co-management process. Consequently, projects that endeavor to weave together TK and WS not only require research protocols that guide this challenging collaboration process (Johnson et al. 2016), but also need to factor in the implications of the knowledge generation process on knowledge systems, governance institutions, and the relations of co-management partners.

The objective of this paper is to provide a long-term, dynamic team-based research framework that considers the different forms of TK, how these may be impacted by knowledge integration processes, and how WS and TK can co-evolve while generating new knowledge that is directed to a collective goal. The latter should be directly linked to the governance and interpretation of knowledge and its implications for co-management and a transition to selfdetermination. Within the context of this paper we define WS as data-driven, empirical research based on reductionist methods and quantitative observations. We take the processes involved in knowledge co-production and place them in the context of long-term application by including co-management and empowerment as central outcomes of the research method. We chose to term the entirety of this process "knowledge coevolution" to highlight the necessary reciprocal changes in knowledge, interpretations, objectives, and outcomes that occur throughout this process and 
that dynamically impact both types of knowledge systems. We also discuss the potential negative effects of WS influence on cultural resilience through knowledge integration practices. Within this paper we use Traditional Knowledge (TK) to describe the broad scope of fact claims from any Indigenous group, and Traditional Ecological Knowledge (TEK) when such claims have specific links with ecological data as interpreted from a Western science (WS) framework. We recognize that Indigenous knowledge systems and sciences are complex and extend far beyond the definition of "traditional", however TK is the current vernacular used in federal law in Canada, such as the Canadian Environmental Assessment Act (2012). We use the term Indigenous with reference to Inuit, First Nations and Métis as defined in the Canadian Constitution.

\section{Knowledge systems, knowledge claims, and knowledge co-production}

\section{Knowledge systems}

Like WS, TK is verified through empirical observations and verification through repetition (Barnhardt and Kawagley 2005), however the reliance on oral traditions, spiritual or holistic views of nature, and inherent trust in ancestor's knowledge is in contrast to the reductionist approach of WS that is based on skepticism and isolation of phenomena (Dale and Armitage 2011; Kovach 2010). TK is gained by experience, transferred among individuals (especially from elders to youth) by stories and direct teachings, and may evolve through experience and observations. While it has been argued that Indigenous knowledge and other knowledge frameworks should remain separate to maintain their integrity (Kimmerer 2013), only interacting when there is clear benefit for each party but otherwise existing in isolation, others suggest that the respectful blending of knowledge types is a vital step in the ultimate survival and well-being of Indigenous communities (Bartlett et al. 2012; Lowan-Trudeau 2012). To function within current governance structures within Canada, it is not practical nor reasonable to assume management methods developed using only one knowledge type will be effective and acceptable. Attempting to maintain complete isolation of TK systems within the context of research and resource management creates logistical barriers to any policy creation and formalization of management regimes. Given the current structure of natural resource management in Canada with a clear transition to co-management in a multi-governance system, blending TK and WS practices becomes a necessity (Dale and Armitage 2011). The challenge will be to do this in a way that strengthens the path to self-determination and that does not sacrifice the preservation of TK and its associated cultural identity and transfer of knowledge.
For research and management purposes, defining what type of knowledge claim constitutes TK and the degree with which certain claims are suitable for use in research or policy implementation is an ongoing challenge (Usher 2000). Classifying and/or categorizing TK claims can help mitigate such challenges. Usher (2000) created a systematic classification system for TEK that distinguishes four knowledge categories based on the level a claim is embedded in cultural practices and knowledge systems; categories one and two are based on factual knowledge from the past and empirical observations of the environment at present and over time as experienced by individuals or reported by the community. categories three and four are broader in scope and encompass aspects of societal norms and knowledge systems specific to a community; category three knowledge includes moral and ethical values of how to interact with the environment, and category four constitutes the foundation of the knowledge system, acting as the lens through which knowledge is interpreted. This framework provides team members a basis to organize and adjudicate knowledge claims from WS and TK and highlights the necessity to scrutinize all knowledge claims and biases equally through the presence of knowledge holders from each knowledge system.

While a useful tool for the collection and systematic digestion of knowledge, Usher does not highlight the importance of collaborative knowledge co-production, discussing the framework in the context of TEK recording for application in WS research or for environmental impact assessment (Usher 2000). When the community has a vested interest, which we argue is predominantly the case or should be the focus of collaborative research objectives, category three and four knowledge must be included throughout the research.

\section{Knowledge co-production-a process of mutual respect}

For knowledge co-production to be effectively executed, participants must be willing to engage in open-mindedness and learning. Even when treated with the utmost respect, documentation and use of TK outside of the control of the knowledge holder(s) creates an innate power imbalance that undermines the advancement of TK holder's rights for selfdetermination. The way that information is documented, synthesized and re-represented will be done in the conventions typical of the academic or management body, potentially contorting the TK (Barber and Jackson 2015).

The practice of "two-eyed seeing" was developed to prevent such appropriation by outlining a process for combining the strengths of Indigenous knowledge and Western knowledge for the benefit of all (Bartlett et al. 2012). A central tenet of this method is that participants from both sides are expected to weigh the perspectives brought forth from each knowledge system equally in terms of their validity 
and importance, and practice introspection to identify personal biases (Martin 2012). This framework has also been extended to "Three-eyed-seeing" or "multi-eyed-seeing" to reflect the potential for it to be applied to numerous cultural perspectives rather than remaining specific to Western science and Indigenous knowledge holders. This method has been employed primarily in health and wellness research (e.g., Marsh et al. 2015; Hovey et al. 2017), but has direct relevance for all collaborative research projects. Similarly, Métissage in research - the blending of Indigenous ways of knowing and research - as presented by Donald (2012) and expanded upon by Lowan-Trudeau (2012) describes a research process that weaves together Indigenous contexts, such as place-based stories (Donald 2012). Métissage characteristics important for respectful and effective knowledge co-production in research include reciprocal benefits, participant review of the data, and maintaining a focus on respect for Indigenous knowledge processes throughout the research. These methodologies provide excellent background and guidance for researchers but do not overtly provide a direct framework for implementation.

For researchers working with TEK, incorporating concepts of social learning theory with defined knowledge categories further enables us to investigate the theoretical characteristics of an iterative and holistic knowledge "coevolution" process, where perspectives, objectives, and cultural norms adapt to account for knowledge obtained during investigation. The extent to which new knowledge is embraced and incorporated into societal adaptation is referred to as "learning loops" or different categories of feedback loops (Armitage et al. 2008). These loops represent the breadth of impact the new knowledge has on behavior, assessment, management practice changes, worldviews, and community culture. Single loop learning adjusts errors through the research routine; for example, modifying harvest strategies. Double-loop learning goes further as it updates management goals and adjusts normative rules and policies. In triple-loop learning processes fundamental governance norms and protocols are addressed and changed. Understanding and anticipating the path of information generated during the research process, and how such knowledge may interact with cultural norms, can transform how researchers and collaborators interact with new knowledge.

There are fundamental challenges with a collaborative knowledge research process for many Indigenous partners. First, TK often includes values, morals, and beliefs while WS seeks to separate knowledge from such values. More often, however, values and underlying norms in WS are simply not explicitly stated, and the consequent impacts of scientific results on the management and governance of ecosystems is not deliberated. Secondly, most Indigenous knowledge systems are directly linked to governance and stewardship but not through a multi-level governance system. New co-management frameworks, like the one recently introduced in Nunavut through the land claims agreement are, therefore, alien to many Indigenous knowledge and governance systems. For example, community Hunting and Trapping Organizations are supposed to feed information into this governance framework but often do not have adequate resources and training to translate their knowledge, and to document and communicate in the language accepted by Western governance institutions (Suluk and Blakney 2008).

Finally, researchers participating in knowledge co-production must acknowledge the inherent power dynamics associated with the institutional hierarchy of research, as well as political factors that influence how information is used (Nadasdy 1999, 2003). If TK holders are not treated equitably, factors may inadvertently encourage the adoption of non-indigenous knowledge systems in order to be heard. This power imbalance can manifest in a number of ways, including but not limited to:

Lack of equity in weight of knowledge types and fact claims.

TK holders not getting to reap the benefit of sharing their knowledge.

TK holders not receiving real gratitude from scientists (little positive feedback).

TK holders not contributing in a real and meaningful way to the application of research.

Observing these phenomena may lead to the degradation of Indigenous culture through the research process by forcing TK holders to adhere to science norms to fully participate.

\section{A new paradigm: knowledge coevolution}

Knowledge co-production highlights the necessity for researchers and TK holders to work together to generate knowledge relevant for both the community and researchers. Consequently, focus is given to deconstructing the power dynamics associated with current Western science research structures and norms.

The route to self-determination requires decolonization that creates an understanding and appreciation of the culturally embedded worldviews, ways of learning and theoretical frameworks of peoples (Smith 1999). Cross-cultural studies of the characteristics of traditional knowledge and science that was carried out in Latin America, Africa and India under the COMPAS (Comparing and Supporting Endogenous development) program concluded that a more culturesensitive approach was needed to succeed in developmental 


\section{Knowledge Coevolution Framework}

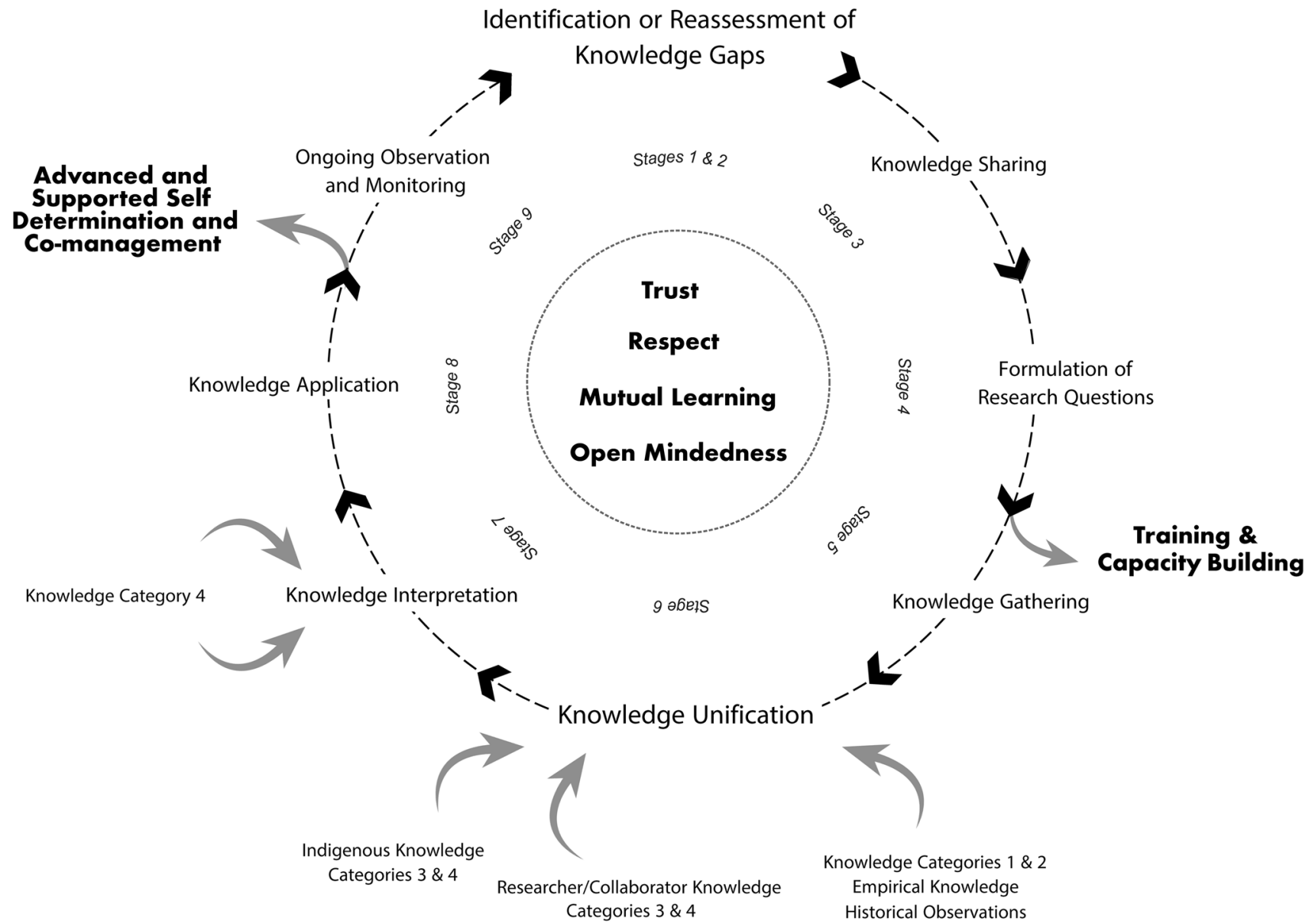

Fig. 1 Conceptual model of the Knowledge Coevolution Framework. Stages correspond to in text descriptions and examples

goals (Havervourt and Reinjtes 2013). They referred to this approach as endogenous development and emphasized that it required different forms of knowledge that do not develop in parallel or merely complement each other but that co-evolve on the basis of their own dynamics and in response to their interaction with other forms of knowledge (Havervourt and Reinjtes 2013).

Here, we present a knowledge coevolution framework that builds on the current body of literature addressing knowledge co-production in the context of WS and research and adds a more formalized objective of capacity building, empowerment, and self-determination as strategic endpoints of the research process. Most importantly knowledge coevolution should work to strengthen TK and not focus on changing or merging TK with WS methods. Knowledge coevolution allows both knowledge systems to advance and evolve on their own and generates new knowledge for the benefit of Indigenous stakeholders by generating data that is meaningful for decision-making and participation of Indigenous peoples in the governance of their lives and the ecosystems they rely on.

\section{Knowledge coevolution research framework}

Research frameworks provide guidance and ensure comprehensive research methodologies and thorough execution of often extremely complex scenarios. Adhering to, or taking guidance from, a research framework can ensure that the various knowledge forms generated from the research achieve the intended goals. Our framework is built on the foundations provided by the literature on Traditional Ecological Knowledge use, knowledge systems, knowledge mobilization, and knowledge co-production, some of which has been reviewed above. This framework is meant to be malleable and dynamic such that it can be applied to research in various disciplines and their collaborations with various user groups, however a major focus is on the empowerment and rights to self-determination of Indigenous peoples and the 
advancement of blended and separate knowledge systems. Indeed, just as there is a spectrum of perspectives among Indigenous communities that are relevant to preserve, WS disciplines also bring unique characteristics that are integral to preserve in certain transdisciplinary projects. This is of particular importance when scientific disciplines have different underlying norms and priorities (e.g., social science and physical geography). The knowledge coevolution framework strives to provide mechanisms for different knowledge types to come together within the context of research regardless of the degree of disparity among the knowledge systems. Doing so ensures a holistic approach and that the focus is not on how to take TK and absorb it in to WS, but how to take a diversity of knowledge systems and scrutinize their application, relevance, and cultural sustainability. We have created a holistic framework diagram (see Fig. 1) and have identified a number of guiding principles and subsequent steps to execute the framework.

A coevolutionary research project adheres to the following principles:

1. Unified and agreed upon research objectives (that might change throughout the process).

2. A governance structure and steering committee for the research and learning process.

3. Emphasis on capacity building in all parties, for example TK (e.g., elder-youth knowledge transfer) and WS (e.g., connecting science more closely to adaptive comanagement).

4. Effective sharing and collective interpretation of knowledge and data analysis.

5. Where multiple knowledge systems co-exist, one does not dominate over others.

6. Beneficial outcomes that empower TK holders and advances their prosperity (as it is self-defined).

7. Enhancement of collective learning, governance and management practices.

\section{Stage 1: origination of the collaboration and defining collective research interests}

The process begins when a party, either the Indigenous community or research group, identifies an issue, an economic opportunity, a governance vacuum or a knowledge gap. In cases where the research need is identified by researchers, knowledge coevolution requires that the research objectives include aspects that are relevant to the lives and concerns of the community members in which the research occurs. An example of the latter is the genetic differentiation of populations of fish (see Schott et al. in review). Just as it would be inappropriate for a community to approach researchers lacking expertise that address their concern, it is inappropriate for researchers to impose research questions upon communities that do not address their expertise or concerns. However, it is very much worthwhile for researchers interested in aspects beyond the scope of typical community concerns, such as more theoretical research, to reach out and assess how they may be able to work with community members to find ways that community priorities can be meaningfully woven into the research project. For example, a researcher working on permafrost measurements may find that nearby communities are concerned about changes in the landscapes that are affecting their travel routes.

A central objective associated with this knowledge gap is required to guide this process. Knowledge unification means that multiple angles of investigative approaches and interpretations are accounted for through the research. As such, the central objective should be shared, though the methodology used to approach the objective can be unique for each group. Consequently, maintaining organization and clearly defined roles and responsibilities is a major challenge.

\section{Stage 2: a governance structure that guides the research, the findings, and potential implementations}

To maintain a streamlined, respectful, and coherent process in large projects, a governance board of representative of researchers, Indigenous groups, and anticipated end users may be necessary. The board of governor's initial responsibility is to create and agree upon a governance structure, ensuring roles and responsibilities are explicitly agreed upon by all parties. Members of the board of governors should be selected by each collaborative group and have experience working in interdisciplinary settings with a diversity of knowledge holders and backgrounds. In cases where collaborators do not have experience of this kind, actively seeking guidance from experienced researchers can overcome this deficit.

Having a pre-determined governance structure ensures continuity and accountability throughout the research project. Regular meetings amongst the board and with necessary stakeholders reaffirms project objectives, maintains communication, and ensures all groups have fair and even access to information such as preliminary results or challenges preventing progress. Focus must be given on maintaining open-mindedness and effective communication amongst the key groups affected by the project. While there is potential for governance to slow progress, a clear and concise plan can compensate for this by improving the group's ability to address project-wide challenges. This does not prevent a project from maintaining flexibility; changes to research objectives should be anticipated and built in to the decision-making process.

Several important "checks" should be completed at predetermined milestones to ensure the project is adhering to the ethical standards determined by the board of governors 
(e.g., reporting) or impasses (e.g., refinement of research plan). While the criteria will vary depending on the project, feedback questions should address knowledge appropriation and power imbalances within the project. For example, "Is there currently a power imbalance with respect to project decision-making?", or "Are knowledge holders comfortable with the way their knowledge has been interpreted and portrayed?". If the answers to these types of questions create conflict or unease, it will force the group to re-evaluate and search for solutions, improving the project and the governance process that will follow or is linked to the research process itself. Even in cases where these issues cannot be eradicated due to logistical or practical constraints, for example research institutions holding research funds, recognizing and discussing their implications and how to accommodate limitations will improve the knowledge coevolution process. These predetermined assessment points are also necessary to assess the overall success of implementing a knowledge coevolutionary research framework; in cases where parties are continually dissatisfied, or similar problems continue to arise, critically assessing the underlying cause and working to address systemic issues may be necessary to facilitate meaningful progress.

\section{Stage 3: knowledge sharing}

Prior to collecting new information, an initial phase of knowledge sharing should be executed. This provides the foundation of understanding from which the research methods and project timelines are determined, and provides an additional opportunity to engage additional collaborators if it is deemed necessary. Uniting fact claims stemming from unique knowledge systems is a challenge that directly and indirectly influences knowledge mobilization across disciplines. Respect and trust are central tenants of effective knowledge transfer (Bartlett et al. 2012), and the flow of information between WS to TK holders requires each group to frame their knowledge in a way that is accessible to the other. Consequently, we pose that this knowledge sharing should occur in person, preferably within the region where the research would be occurring in relatively frequent intervals ${ }^{1}$. Initial knowledge sharing should occur in a manner that respects the integrity of each knowledge system through healthy discourse. There are a variety of formalized methods to bridge knowledge systems, including participatory mapping, workshops, and scenario planning. ${ }^{2}$ Researchers

\footnotetext{
1 The frequency will depend on cultural norms. For example, some Indigenous communities are more accustomed to oral transfer of information, which makes face-to-face interactions and relationship building a crucially important component of research methods.

2 A comprehensive list of such methods is presented in Johnson et al. (2016)
}

should be willing to break the status quo of formal emails and written proposals, and instead speak directly with knowledge holders in the community, out on the land, and in a manner that is comfortable for them.

The initial knowledge sharing often presents a challenge, as personal biases associated with cultural identities mediate the interpretation of fact claims; what one group sees as a potential explanation for a given phenomena is innately rooted in deeper cultural belief systems (e.g., knowledge categories 3 and 4). This is particularly challenging when aspects of spirituality or religious beliefs influence the interpretation of fact claims. Open-mindedness, listening, patience and respect are crucial elements required for respectful and effective knowledge sharing to occur. Being comfortable and accepting of differing opinions and interpretations is important in any good teambased research, yet when bridging knowledge systems, it is a critical necessity.

\section{Stage 4: formulation of research objectives}

Based on the knowledge gaps identified during the knowledge sharing stage, the research team should now have the information necessary to identify shared research objectives. We argue that these research objectives must address knowledge gaps that are meaningful for all parties and fall within the expertise of the research team. Identifying and involving the appropriate TK holders should be determined by the Indigenous representatives, or through the mechanisms instructed by the community provided research protocols if available.

It is reasonable to assume that some unanticipated knowledge gaps may be identified during the initial knowledge sharing phase and may require a reassessment of the collective research interests. This is part of the reciprocal and iterative nature of knowledge coevolution and should be viewed as an opportunity for refinement rather than be avoided. It is possible that addressing these questions may fall outside of the research team's expertise. Consequently, we suggest that the (re)formulation of research questions should take place over the course of at least two, if not more face-to-face meetings (video or conference calls a second-best) that allow new collaborators and perspectives to be added to the project if deemed necessary to adequately address the new research objectives. It is also important to moderate expectations in this case, as both TK holders and researchers may not be able to address all knowledge gaps within the constraints of the current project, and it may not be possible to bring in the appropriate people in a timely manner. Maintaining open and respectful communication about these aspects of the project is critical to ensuring all parties have realistic and feasible expectations for project objectives and outcomes. 


\section{Stage 5: knowledge gathering}

\section{Research methodologies}

Knowledge gathering is the phase where new information is collected to facilitate investigation of the project objectives. Scientific methodologies for knowledge gathering are discipline and context specific, and will not be covered here. Considerations for scientific methods that are required for knowledge coevolution are:

1. All team members have full understanding of research methods and agree they are the best way to address the study objectives within project limitations (e.g., budget, logistics).

2. Method limitations are clearly outlined and understood by all parties involved.

3. Research methods do not violate cultural beliefs and are culturally appropriate.

4. Anticipated timelines for knowledge collection are discussed and agreed upon by all team members.

While these requirements appear straight forward, unexpected barriers may arise. For example, research associated with culturally important species or landmarks presents a suite of unique challenges. Sampling methods that are common place among researchers may catch Indigenous collaborators off guard, particularly if the technology has not been described and demonstrated in an accessible manner. As a result, researchers must be prepared to go through their data collection plans in detail, including lay summaries, demonstrations, and examples of other regions using the same methods.

If parties cannot agree upon a methodology, e.g., the researcher's preferred method violates a cultural belief, an opportunity for ingenuity may arise. Understanding what aspects of the method are inappropriate can lead to refinement or addition of steps that better encompass the culture of the Indigenous communities. Developing novel techniques together improves knowledge coevolution and further empowers community members. This has been observed in wildlife studies, where researchers capture and attach electronic tracking devices to animals and release them back in to the wild. In one case, previous tagging methods developed for use on beluga whales involved netting and landing the whales in shallow coastal waters. This method was determined to be too invasive and an inappropriate way to interact with wildlife by the Inuit communities in the Inuvialuit Settlement Region. In response, the research group created a new tagging method based on traditional hunting equipment and techniques and did not require the whale to be captured, developed with guidance from the project steering committee (Loseto et al. 2018). Not only did the method better adhere to cultural practices and better involve community members compared to the past, researchers are now able to tag whales in areas they were previously not able to, such as deeper waters, due to the skill and precision of Inuit harvesters.

\section{Capacity building}

Here we refer to the process of engaged collaboration with and providing training for Indigenous partners as "capacity building". This occurs directly when individuals join the research team and are trained and/or hired to assist with the project, and inadvertently, through ongoing exposure to the processes and requirements associated with running a research program. Unique to our knowledge coevolution model, however, is the pointed effort to strengthen TK itself during capacity building. For example, community guided TEK workshops can facilitate the appropriate documentation of TK and engagement of different knowledge holder groups. An enhanced knowledge transfer process among participants should be strived for if of interest to the community. To execute this properly, the appropriate TK holders must take the lead where appropriate, while WS researchers reflect on their own principles, their limitations, and merits. Often an interdisciplinary approach is required that connects WS principles and methods, and links them more effectively and appropriately to TK. Timing for each group's leadership role can be formally established by the project board of governors, or occur organically during training or knowledge gathering activities.

An aspect of capacity building that is inherent to knowledge coevolution is the inclusion of Indigenous knowledge holders in all aspects of the research process. Consequently, "training and capacity building" for project management occurs through experience and guidance from those more familiar with the administrative and technical requirements of formal investigative inquiry. This practice is one of many ways that ethically conducted research can provide a platform for empowerment and progress towards self-determination and co-management in a way that adheres to the current requirements of the Western governance regime currently present in Canada. While perhaps not ideal, for progress to occur in a timely manner, progress within the current system must be made.

It is again crucial to ensure that methods used for capacity building are suited to the intended audience when possible. For example, many communities may suggest that teaching should be done in a manner that departs from colonial norms (e.g., heavy reliance on lecturing and written instruction; McGregor 2012) and is imbedded in other culturally relevant activities such as story telling and feasts (Iwama et al. 2009). The training should not assume or impose specific qualities 
common in Western culture and should ultimately be guided by Indigenous team members. Experiential learning, for example, is often more successful in Indigenous communities but is often overlooked in southern training practices (Bartlett et al. 2012). A good example are elder-youth workshops carried out on the land where both elders and youth learn from each other while engaging in important cultural and food provision activities that are crucial to indigenous survival and livelihoods (Wolfe et al. 2018).

\section{Stage 6: knowledge unification-new knowledge creation that stands on its own and is useful to end users}

The process of bringing knowledge together while maintaining the integrity of both knowledge systems in a cohesive and applicable way is perhaps the most complex component of knowledge coevolution. The spirituality imbedded in Indigenous ways of knowing in contrast with the logicdriven principles of WS can create ideological mismatch, as described previously. Through the research process up until this point (knowledge sharing, identifying joint objectives, ensuring methodologies are appropriate, training and capacity building for unified information gathering) many parties have played a role in generating knowledge that addresses the specific research objectives together. The participatory and collaborative nature of the knowledge coevolutionary process is designed to help team members become acquainted with each knowledge system, and thus more amenable to alternative knowledge types. Indeed threading together the story from multiple sources of information and perspectives facilitates interpretation and allows each group to understand the perspectives of each audience.

The new knowledge created must be translated to a form that is accessible by all team members. If a steering committee has been created as part of the project governance structure, it can be used to aid in this process. Communicating research results can be a particularly daunting challenge when results are extremely technical or require a great deal of contextual understanding. Some of this information should be addressed during the formulation of research questions and determining appropriate research methods stage. If this is done properly, team members should be somewhat primed. The knowledge coevolution process requires more focus on this stage than typical research projects and as such additional time should be incorporated for project timelines to ensure the process is not rushed. At this stage the potential impacts of the results on social values, norms, environmental stewardship and the way socio-ecological interactions are governed need to be discussed in order to have a meaningful transition into knowledge interpretation and application.

\section{Stage 7: knowledge interpretation}

Interpretation of the results is communicating what the research results mean from the perspective of the individual, seated within the context of their knowledge system and moral and ethical beliefs (i.e., knowledge categories 3 and 4; Denzau and North 1994). This process must include the perspectives of those who contributed TK during the knowledge gathering phase when possible. This can be done through group workshops or follow-up meetings. Care must be taken to avoid imposing the interpretations and perspectives of others upon those who are not a core member of the research team but who have had their knowledge included as part of the study.

The way that these interpretations are communicated amongst the group must again be done in a manner that is respectful and accessible for all parties, similar to what was required for the knowledge unification process. Here, however, alternative interpretations may be presented. Reasons for such differences may be vast, and can stem from something as simple as misinterpretation due to poor communication of results, or as complex as stemming from differences in worldviews. In the latter case, researchers should be encouraged to re-assess their interpretations with respect to how their understanding of science fits within the more holistic context of the relationship between people and the environment. We argue that to move towards culturally relevant co-management, flexibility and ingenuity should be incorporated more so with WS interpretations than the Indigenous team members. The reason for this is that indigenous knowledge systems are already integrated between normative and positive knowledge components and wholistic in nature, while WS is compartmentalized.

In cases where conflicting interpretations of results occur that is not easily rectified through open discussion, the team must scrutinize the evidence together and weigh the ultimate ramifications of alternative interpretations. Care must be taken not to allow conflict to escalate by making concerted effort to better understand the similarities and differences in worldviews underpinning the disagreement (Nguyen et al. 2016). Because self-determination is a central objective of knowledge coevolution, Western scientists may need to consider mitigating their perceived risk of the communities' interpretation through open dialogue, deliberation, and adaptive co-management approaches. Indigenous decisions are usually based on consensus which requires lots of discussions, weighing of options and implications until all sides are content with moving forward and making a decision (even if not everybody agrees). For example, in cases where the results affect resource management choices, openly discussing what each party believes will be the outcome of alternative management strategies on the environment, socioeconomic opportunity, and political environment is crucial. This should be done in a manner that is cognizant of power 
imbalances and potential persuasion, and the ultimate goal of movement towards self-determination.

\section{Final stages: knowledge application, ongoing observation and monitoring}

The scale at which knowledge obtained through the research project can be applied varies depending on the context of the research project, yet the knowledge coevolution process itself aims to facilitate the deconstruction and redistribution of benefits associated with research and this can be realized in the way that knowledge application occurs. It is important to point out here that the top-down management practices that have been regulating Indigenous communities are often in direct violation with their rights, and are not likely to persist in perpetuity. Consequently, priority should be given to determining culturally appropriate management strategies founded in evidence gathered by teams that involve Indigenous peoples in a meaningful way.

Whether or not the research can be directly applied to management does not limit the ability for a research project to positively improve self-governance for Indigenous peoples. The research process often uncovers opportunity for ongoing observation and monitoring, or identifies additional knowledge gaps that require further investigation. If the knowledge coevolution framework is followed, community members within the project team should now have many of the skills required to address these needs. Consequently, ongoing observation and monitoring is something that can be achieved with as little intervention as possible, and could be used as local employment opportunities for biomonitoring, fishery and wildlife assessment. Researchers can move to a more supportive role if necessary, assisting with the acquisition of funding or additional training opportunities.

\section{Application of our framework to a harvest study process}

Our suggested framework can be applied to various research and resource management and governance scenarios such as fishery sampling, assessment, and co-management (Schott et al. in review). Below we will provide an example where each stage of the knowledge coevolution framework is illustrated.

\section{Stages 1 and 2: defining collective research interests}

This framework evolved through a research project on commercial fisheries and food security in the community of Gjoa Haven, Nunavut. The project stemmed from the desire to develop commercial fisheries in the community. Representatives of the Hunters and Trappers' Association (HTA) reached out to Peter van Coeverden de Groot, a biologist that had been working in the community for several years, requesting research support. The potential for genomic tools to be applied to fisheries in this region to support the community's research objectives was evident. This led to a Genome Canada application that was written in collaboration with four principal investigators and the HTA. ${ }^{3}$ This project was funded and includes a large Genomics Environmental, Ethical, Economic, Legal, and Social (GE $\left.{ }^{3} \mathrm{LS}\right)$ component led by S. Schott. The project governance structure was determined early on to consist of a knowledge translation advisory board, quarterly special HTA meetings, annual general project meetings, and fishery advisory board meetings (Stage 2). See Schott et al. (in review) for further details.

\section{Stages 3 and 4: knowledge sharing and formulation of research objectives}

The project started with a TEK workshop in February 2016 that brought together researchers and community members to share knowledge of historic and current fishing practices and species distributions. Follow-up workshops in August 2016 validated this information and identified the need for a more detailed harvest study due to knowledge gaps regarding the specific locations of contemporary harvesting, overall cost of harvest, and how these interact to influence community food security.

\section{Inherent flexibility and iterative research process}

The first year of the project focused primarily on subsistence fishing sites, potential commercial fishing sites, and food security in the community. Through this work, additional research questions were identified that required a more detailed and locally involved harvest study to reach community objectives. Specifically, more detailed information regarding the cost of hunting and fishing, spatio-temporal variations in harvesting, and productivity (i.e., catch-perunit-effort or CPUE) from different sites. In August 2016, a draft harvest study was developed that consisted of three components: a registration survey, the tracking of hunters using remote satellite devices, and a post-trip harvest, and economic survey with a representative sample of hunters. Working directly with the HTA through the Knowledge Translation Advisory Board and special HTA meetings, a pilot of the study methods was run in August/September 2016. Insights from this pilot were directly integrated into a revised version of the survey. The HTA officially approved

\footnotetext{
${ }^{3}$ For further details on the specific timelines and objectives of the research process, see Schott et al. (in review).
} 
the harvest study method and survey questions in December 2016 after several revisions of questions in the fall of 2016.

Given the extension of research objectives beyond the scope of the Genome Canada grant, additional funds were required to achieve the new research goals. This led to a substantial stand-alone project that was applied for through Polar Knowledge Canada in January 2017 and was funded in May 2017. Subsequently, community member James Qitsualik piloted the study again without the presence of researchers to identify the real practicality of the study design and satellite devices. Immediate feedback revealed that the chosen satellite device did not suit the needs of harvesters as it did not function as a GPS with mapping capabilities, and communication was cumbersome due to awkward keystrokes (i.e., there was no keyboard). Piloters indicated they would not use the device to report harvests.

Lack of uptake from the harvesters required an upgrade of the satellite device to a newer model that included mapping capabilities, and innovative solutions to make the reporting of harvests and observations easier. Working with the Geomatics and Cartography Research Centre at Carleton University and the satellite GPS developers, a user-friendly, harvest study-specific application was created that captured the required information without the need for harvesters to enter the data directly by using drop-down selection menus. The entered data automatically uploads to a community-specific harvest Atlas. The new device was piloted in August 2017 by the same hunter and collaborator, James Qitsualik, and was approved. The study was officially launched in late August 2017.

\section{Training and capacity building}

While the final piloting and approval of study design was taking place, hiring and training of a harvest study facilitator and youth apprentice occurred. The study facilitator administers the study by signing out the tracking devices, collecting surveys, and training hunters on the use of satellite devices. Together with the HTA, the facilitator is responsible for ensuring that the participating harvesters are a representative sample of the type of harvesters in the community (e.g., frequent, occasional). Capacity building is ongoing and includes training in data stewardship, reporting, presentations, meetings, workshops, mapping, and peer-topeer training.

\section{Stage 5: knowledge gathering}

Data collection began in August 2017 and is currently ongoing. During this time, issues regarding study participation and the clarity of project objectives, study methodology, and timelines became apparent. Initially, there was limited participation in the harvest study. To better understand the reasons for this, participant workshops and individual follow-up surveys were used to understand the reluctance to take out the tracking devices. From these efforts, it became apparent that the objectives of the study and how the outcomes would benefit the community were unclear to participants. Uptake began to increase only when individual participants that recognized the benefit of the information generated by the project started sharing the advantages of taking out the satellite device with others. In particular, the improved mapping, communication capabilities, and SOS feature of the tracking device was popular among participants. Workshops were held bi-annually with harvest study participants to demonstrate how the data was being accrued in the Atlas and give participants the opportunity to provide feedback. As more data was visually displayed on the harvest study Atlas, hunters could see how the information they enter on the device while on the land was recorded and visually summarized. These bi-annual update workshops allowed participants to better understand the study objectives, why the study methods were chosen, and envision how the data could be used in the community. For researchers, these workshops provided valuable feedback from the users that improved software design, visual display, and refinement of study facilitation to make it easier for the participants (e.g., rewording survey questions, time frames to pick up GPS devices).

\section{Stage 6: knowledge unification}

The harvest study objectives are founded in aspects of WS including conservation, socio-economics, and anthropology, as well as Inuit knowledge of culture, social norms, and the environment. By bringing the two knowledge types together from the beginning of the project, we are positioned to separately inform both knowledge systems independently and produce new knowledge. With respect to the harvest study, new knowledge that meets the agreed upon study objectives includes the evaluation of cost of hunting in different locations, cost and distribution of food in the community, and the verification of the safety of travel routes. The Western science interest was wanting to know how much it cost to provide food, and how much harvesting of different animals occurs, while the TK was more focused on the cost of hunting and success rates in different locations. The primary focus from the Inuit perspective was to also record and understand the dangers associated with travel on the land, as travel routes and conditions have changed dramatically since elders lived on the land and established safe historical travel routes. This information feeds back in to Western science objectives of understanding how climate change has impacted the Northern environment, while for Inuit this investigation opens a dialogue between youth and elders about changes on the land and new, safe travel routes. 


\section{Stages 7 and 8: knowledge interpretation and application}

Interpretation of the harvest study data occurs during workshops, special meetings, and conferences with registered hunters, the HTA board, the harvest study facilitator and youth apprentice, and other HTAs. Results from the study are presented and group discussion occurs on the validity of the results, additional research questions and objectives, and potential applications (e.g., the cost of hunting compared to the offered compensation through community food distribution programs). Having both parties involved in the interpretation process can also lead to spin-off objectives, in this case a more pointed evaluation of costs, distances travelled, and success rates associated with Polar bear harvests, as well as a study of the food distribution programs in the community. Application of the Atlas to demonstrate Inuit presence and land-use under the context of Northwest Passage Sovereignty was also brought up by those participating in the harvest study. Ongoing research will advise potential governance, management practices, and food support programs in the communities and Northern food subsidy policies in Canada.

\section{Conclusion}

Indigenous knowledge is being lost at an accelerating rate, as is the cultural knowledge systems through which Indigenous knowledge holders interpret the ecological world around them. While perhaps counter-intuitive, researchers are in a unique position to provide the support to achieve self-determination and empowerment, transferring the balance of power away from colonial institutions. At the same time, approaching this complex situation with the intention of preserving Indigenous knowledge and culture can provide a sense of pride and indicate acceptance and understanding in a system that has otherwise served to counter and suppress Indigenous peoples. We argue it is the moral and ethical responsibility of Western scientists working in and with Indigenous communities to adhere to the National Inuit Strategy on Research (2018) by making a concerted effort to uncouple investigative research from colonial based hierarchies and power dynamics. The knowledge coevolution framework provides guidance for achieving this because it emphasizes the reinstatement of Indigenous knowledge systems, which requires understanding and awareness of how they are affected by colonial value and knowledge systems.

Our proposed knowledge coevolution framework distinguishes itself from knowledge coproduction because it directly addresses power imbalances and strives to create a space and route for self -determination. Its emphasis on strengthening traditional knowledge transfer among community members, and providing training and practical experience in research, governance, and monitoring necessary for meaningful co-management is essential for the transition to an independent management system. Our proposed framework is based on mutually advantageous research objectives that strengthen traditional and local knowledge while invigorating Western science by connecting it with its routes of ethics and normative science, which has arguably been forgotten along the way. This framework positions scientists to increase the uptake of research results by Indigenous partners and the wider policy environment, a phenomena that is often lacking in today's research culture that is too focused on unidirectional communication and recognition of narrow research achievements.

Acknowledgements We acknowledge the contributions of our Inuit collaborators who declined to co-author this theoretical framework manuscript. We respect our colleague's wishes to instead focus on a forthcoming paper outlining the detailed methods of the Towards a Sustainable Fishery for Nunavummiut research project. We thus express our gratitude to James Qitsualik and Simon Okpakok for their insightful comments which greatly improved the structure of our framework. We also thank Jacob Keanik, Allen, Helen Kaloon, Brent Puqiqnaq, and Jordan Takkiruq for their insights, the board of the Gjoa Haven Hunters and Trappers Association, and the community of Gjoa Haven for their ongoing support. We also thank V Walker, PVC de Groot, and S Lougheed. This research was supported by a Genome Canada's Large Scale Applied Research Project (OGI-096) grant and by Polar Knowledge Canada: Knowledge Management and Engagement (PKA1718-0002) funding.

Open Access This article is licensed under a Creative Commons Attribution 4.0 International License, which permits use, sharing, adaptation, distribution and reproduction in any medium or format, as long as you give appropriate credit to the original author(s) and the source, provide a link to the Creative Commons licence, and indicate if changes were made. The images or other third party material in this article are included in the article's Creative Commons licence, unless indicated otherwise in a credit line to the material. If material is not included in the article's Creative Commons licence and your intended use is not permitted by statutory regulation or exceeds the permitted use, you will need to obtain permission directly from the copyright holder. To view a copy of this licence, visit http://creativecommons .org/licenses/by/4.0/.

\section{References}

Abu R, Reed MG, Jardine TD (2019) Using two-eyed seeing to bridge Western science and Indigenous knowledge systems and understand long-term change in the Saskatchewan River Delta. Int J Water Res Dev. https://doi.org/10.1080/07900 627.2018.1558050

Armitage D, Marschke M, Plummer R (2008) Adaptive co-management and the paradox of learning. Glob Environ Change 18:8698. https://doi.org/10.1016/j.gloenvcha.2007.07.002

Armitage DF, Berkes F, Dale A, Kocho-Schellenberg E, Patton E (2011) Co-management and the co-production of knowledge: learning to adapt in Canada's Arctic. Glob Environ Change 21:995-1004. https://doi.org/10.1016/j.gloenvcha.2011.04.006 
Barber M, Jackson S (2015) Knowledge making: Issues in modeling local and Indigenous ecological knowledge. Hum Ecol 43(1):119-130

Barnhardt R, Kawagley AO (2005) Indigenous knowledge systems and Alaska Native ways of knowing. Anth Ed Q 36(1):8-23

Bartlett C, Marshall M, Marshall A (2012) Two-eyed seeing and other lessons learned within a co- learning journey of bringing together Indigenous and mainstream knowledges and ways of knowing. J Environ Stud Sci 2(4):331-340. https://doi.org/10.1007/s1341 2-012-0086-8

Berkes F (2009) Evolution of co-management: role of knowledge generation, bridging organizations and social learning. J Environ Manage 90:1692-1702. https://doi.org/10.1016/j.jenvm an.2008.12.001

Boudreau SA, Fanning L (2016) Nunavut fisheries co-management and the role of the Nunavut Land Claims Agreement in fisheries management and decision making. In: Chircop A, Coffen-Smout S, McConnell ML (eds) Ocean Yearbook, vol 30, pp 207-241

Dale A, Armitage D (2011) Marine mammal co-management in Canada's Arctic: knowledge co-production for learning and adaptive capacity. Mar Pol 35(4):440-449. https://doi.org/10.1016/j.marpo 1.2010.10.019

Denzau AT, North DC (1994) Shared mental models: ideologies and institutions. Kyklos 47(1):3-31

Donald D (2012) Indigenous Métissage: a decolonizing research sensibility. Int J Qual St Educ 25(5):533-555

Fisheries and Oceans Canada (2018) Joint statement on the development of fisheries regulations for the Nunavut settlement area. July 9 2018. www.dfo-mpo.gc.ca/media/statements-declarations/nunav ut-eng.htm

Gondor D (2016) Inuit knowledge and environmental assessment in Nunavut, Canada. Sustain Sci 11:153-162

Havervourt B, Reinjtes C (2013) Diversities of knowledge communities, their worldviews and sciences: on the challenges of their co-evolution. In: Subramanian S, Pisupati B (eds) Traditional knowledge in policy and practice: approaches to development and human well-being. United Nations University Press, New York, pp 12-30. https://doi.org/10.18356/f69d7617-en

Hovey RB, Delormier T, McComber AM, Lévesque L, Martin D (2017) Enhancing indigenous health promotion research through two-eyed seeing: a hermeneutic relational process. Qual Health Res 27(9):1278-1287

Inuit Tapiriit Kanatami (ITK) (2018) National Inuit Strategy on Research. ITK, Ottawa, Ontario, p 46

Iwama M, Murdena M, Marshall A, Bartlett C (2009) Two-eyed seeing and the language of healing in community-based research. Can $\mathbf{J}$ Native Educ 32(2):3-23

Johnson JT, Howitt R, Cajete G, Berkes F, Pualani Louis R, Kliskey A (2016) Weaving Indigenous and sustainability sciences to diversity our methods. Sustain Sci 11:1-11. https://doi.org/10.1007/ s11625-015-0349-x

Kimmerer RW (2013) The fortress, the river and the garden: a new metaphor for cultivating mutualistic relationship between scientific and traditional ecological knowledge. In: Kulnieks A, Longboat $\mathrm{D}$, Young K (eds) Contemporary studies in environmental and Indigenous pedagogies. Sense Publishers, Rotterdam, pp 49-76

Kimmerer RW, Lake FK (2001) The role of indigenous burning in land management. J For 99:36-41

Kovach M (2010) Indigenous methodologies. University Press, Toronto
Loseto, L, Arey, D, Benoit, D, O’Corry-Crowe, G, Elias, G, Noksana Sr, J, Felix, JL, Lam, J, Hansen, J, Pokiak, J, Kaglik, L, Day, L, Akhiatak, P, Hussey, N, MacPhee, S. (2018) Co-design of a less invasive method for tagging Eastern Beaufort Sea whales (Delphinapterus leucas). In: Arctic Net Annual General Meeting Ottawa Dec 132018.

Lowan-Trudeau G (2012) Methodological Métissage: an interpretive Indigenous approach to environmental education research. Can J Environ Ed 17:113-130

Marsh TN, Cote-Meek S, Toulouse P, Najavits LM, Young NL (2015) The application of two-eyed seeing decolonizing methodology in qualitative and quantitative research for the treatment of intergenerational trauma and substance use disorders. Int J Qual Methods. https://doi.org/10.1177/1609406915619046

Martin DH (2012) Two-eyed seeing: A framework for understanding Indigenous and non- Indigenous approaches to Indigenous health research. Can J Nurs Res 44(2):20-42

McGregor HE (2012) Curriculum change in Nunavut: towards Inuit Qaujimajatuqangit. McGill J Ed 47(3):285-302

Nadasdy P (1999) The politics of TEK: Power and the "integration" of knowledge. Arctic Anthropol 36(2):1-18

Nadasdy P (2003) Reevaluating the co-management success story. Arctic 56(4):367-380

Nguyen VM, Young N, Hinch SG, Cooke SJ (2016) Getting past the blame game: Convergence and divergence in perceived threats to salmon resources among anglers and indigenous fishers in Canada's lower Fraser River. Ambio 45:591-601

Rathwell KJ, Armitage D, Berkes F (2015) Bridging knowledge systems to enhance governance of the environmental commons: a typology of settings. Int J Commons 9(2):851-880. https://doi. org/10.18352/ijc.584

Roué M, Nakashima D (2002) Knowledge and foresight: the predictive capacity of traditional knowledge applied to environmental assessment. Int Soc Sc J 54(173):337-347

Schott S, Qitsualik J, Okpakok S, Van Coeverden de Groot P, Chapman JM, Lougheed S, Walker V (in review) Operationalizing knowledge coevolution: towards a sustainable fishery for Nunavummiut, Arctic Sc AS-2019-0011.R2

Smith L (1999) Decolonising methodologies: research and indigenous peoples. Zed Books, London

Suluk TK, Blakney SL (2008) Land claims and resistance to the management of harvester activities in Nunavut. Arctic 61:62-70

Tengö M, Brondizio ES, Elmqvist T, Malmer P, Spierenburg M (2014) Connecting diverse knowledge systems for enhanced ecosystem governance: the multiple evidence base approach. Ambio 45(5):579-591

Usher P (2000) Traditional ecological knowledge in environmental assessment and management. Arctic 53:183-193

Von der Porten S, de Loë RE, McGregor D (2016) Incorporating Indigenous Knowledge systems into collaborative governance for water: challenges and opportunities. J Can St 50(1):214-243

Wolfe S, Schott S, Chapman JM (2018) Iqalungmiut: a Gjoa Haven knowledge-sharing workshop; Geological Survey of Canada. Open File. https://doi.org/10.4095/313097

Publisher's Note Springer Nature remains neutral with regard to jurisdictional claims in published maps and institutional affiliations. 